\title{
The neglected face of war surgery: sepsis redo thoracotomy following inadequate first responder management in the Libyan civil war
}

Selçuk Gülmez, Aziz Serkan Senger, Ebubekir Gündeş, Ulaş Aday, Hüseyin Çiyiltepe, Durmuş Ali Çetin, Emre Bozdağ

Department of Gastroenterological Surgery, Kartal Kosuyolu High Education and Research Hospital, Istanbul, Turkey

Submitted: 6 June 2017

Accepted: 16 June 2017

Arch Med Sci Civil Dis 2017; 2: e92-e95

DOI: https://doi.org/10.5114/amscd.2017.68654

Copyright (c) 2017 Termedia \& Banach

Chest injuries account for about 15\% of war injuries [1], and the main cause of wartime mortality and morbidity is chest injuries [2]. The consequences of penetrating thoracic injuries can be serious, and mortality can occur at the scene especially if this situation is a result of a gunshot wound. Mortality can sometimes occur, specifically as a result of sepsis, in the late period. Noting this can make prudent intervention possible during the first response and enable a solution to the problem even before it happens. There might not be enough time, however, for a second intervention for complications related to existing injuries in extraordinary situations like war, and the picture may easily result in mortality.

The 23-year-old male patient had initially received left anterolateral thoracotomy, pneumorrhaphy, and tube drainage procedures in Libya because of a penetrating gunshot wound in the left lung during the civil war. The patient was referred to our clinic with a picture of sepsis on the $17^{\text {th }}$ post-operative day for advanced treatment. The patient's presenting general condition was bad, his consciousness was somnolent, he was hypotensive, tachycardic, and tachypneic with axillary fever of $38.9^{\circ} \mathrm{C}$. $\mathrm{He}$ also had bilaterally extensive subcutaneous emphysema which was more pronounced in the left thorax. Respiratory sounds could not be heard in the left lung through auscultation. The thoracic tube placed in the middle of the current thoracotomy line of the patient was not oscillating at the time of presentation. There was pus at the entry point of the tube into the skin and in the inner wall of the tube. The results of the thoracic x-ray and the thoracic computed tomography (CT) performed by us on the day of the patient's presentation at our clinic revealed left pneumothorax, pneumomediastinum, atelectasis, multilocular $6 \times$ $4.5 \mathrm{~cm}$ intraparenchymal upper left lobe, infected hematoma with air value, segmental fracture in the posterior left $5^{\text {th }}$ rib (Figures $1 \mathrm{~A}, \mathrm{~B}$ ), and extensive pleural effusion. Moreover, metal parts belonging to the gunshot wound were observed in the upper abdominal wall's left lateral part. His laboratory infection parameters were high (C-reactive protein (CRP): $26 \mathrm{mg} / \mathrm{dl}$, leukocytes: 21500/mm³). His drawn arterial blood gas showed signs of hypoxemia $\left(\mathrm{pO}_{2}: 58 \mathrm{~mm} \mathrm{Hg}\right)$ and hypercarbia $\left(\mathrm{pCO}_{2}\right.$ : $43 \mathrm{~mm} \mathrm{Hg}$ ), which suggested pulmonary organ failure. Furthermore, the patient was receiving totally parenteral nutrition (TPN) from the central venous pressure (CVP) catheter. Reoperation was planned from the pa-

\author{
Corresponding author: \\ Selçuk Gülmez \\ Department of \\ Gastroenterological Surgery \\ Kartal Kosuyolu \\ High Education and \\ Research Hospital \\ 34865 Istanbul, Turkey \\ Phone: +90 5530846215 \\ E-mail: selcukgulmez54@ \\ hotmail.com
}



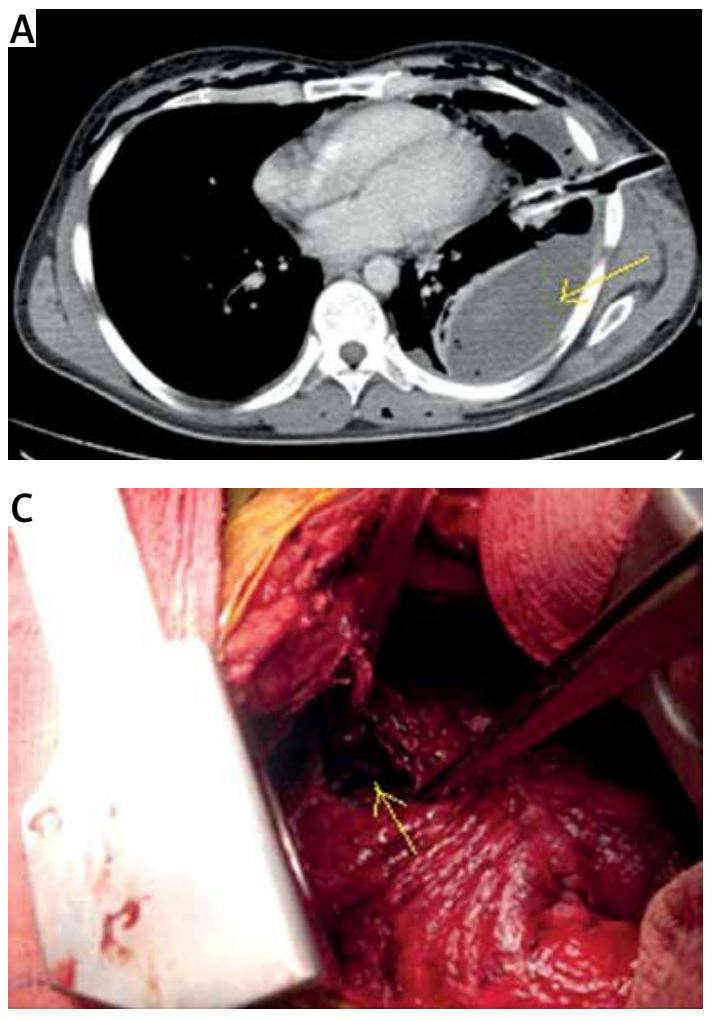

tient's existing thoracotomy because of his infected intraparenchymal pulmonary hematoma possibly causing sepsis, compression atelectasis secondary to collection, pulmonary organ failure as well as collapsed lung, and presence of purulent material in the thoracic tube. Exploration revealed entry and exit holes $2 \times 2 \mathrm{~cm}$ in size from a gunshot wound in the upper left lung lobe's anterior (Figure $1 \mathrm{C}$ ) and posterior parts with hematoma defined by CT inside and intraparenchymal necrotic foci were observed. The extensive fibrin membranes, localized empyema, pleural effusion, and rib fracture pieces were cleaned meticulously; fluid, tissue, and catheter cultures were taken.

The ventilation check performed in order to assess damage control of the left lung showed primary irreparable serious air leak extending from the anterior to the posterior in the gunshot wound area and venous hemorrhaging from multiple foci in the form of intraparenchymal leakage on this line of trajectory. Non-anatomical resection was performed with a stapler in the upper lobe of the lung based on this finding. Other pulmonary areas damaged by rib fractures and maintaining air leak were primarily sutured. A total of two new thoracic tubes were placed from the inferior of the thoracotomy line to the apex $28 \mathrm{~F}$ and basal $32 \mathrm{~F}$. The thoracotomy incision was debrided and primarily closed. Likewise metal foreign bodies which did not penetrate into the abdomen belonging to the gunshot wound were removed and the skin there was debrided. The postoperative X-ray of thorax

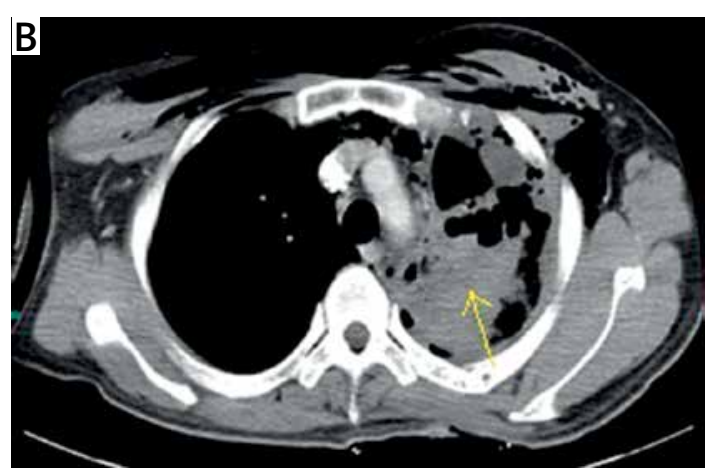

Figure 1. A - Preoperative thorax CT with pleural effusion (arrow). B - Pulmonary hematoma with air values (arrow) and pneumomediastinum. C - Gunshot wound in the upper left lung lobe (arrow)

showed that the findings improved significantly (Figures 2 A, B).

Acinetobacter baumannii reproduced in the catheter cultures and appropriate antibiotic treatment was organized accordingly. The patient was discharged from our clinic with full recovery on the $12^{\text {th }}$ post-operative day.

War surgery necessitates a large and experienced surgical team and a multidisciplinary approach as well as quick thinking and intervention [3], as it poses greater risk of infection in comparison to other injuries [4].

In gunshot wounds, there is a risk of cavitation, hematoma, and secondary contamination alongside the direct damage of the tissues [5]. There are more cases of vital pulmonary parenchymal damage in high-speed gunshot wounds used in wartime, and this situation is related to increased incidence of empyema [1]. Most penetrating chest wounds in both wartime and in civilian life are caused by gunshot wounds, and the factors determining mortality are hemorrhage, respiratory failure, and sepsis [6].

Trauma-related mortality cases can be analyzed in 3 different parts as sudden, early, and late periods. Sepsis caused by an inadequate early response plays the key role in late-term mortality, which may extend from a couple of days to weeks [3]. Endotoxins formed in alveolar hypoxia, which is a natural consequence of such injuries, trigger sepsis [7]. Hemothorax, which is a complication of penetrating chest wounds, or pleural effusion may 

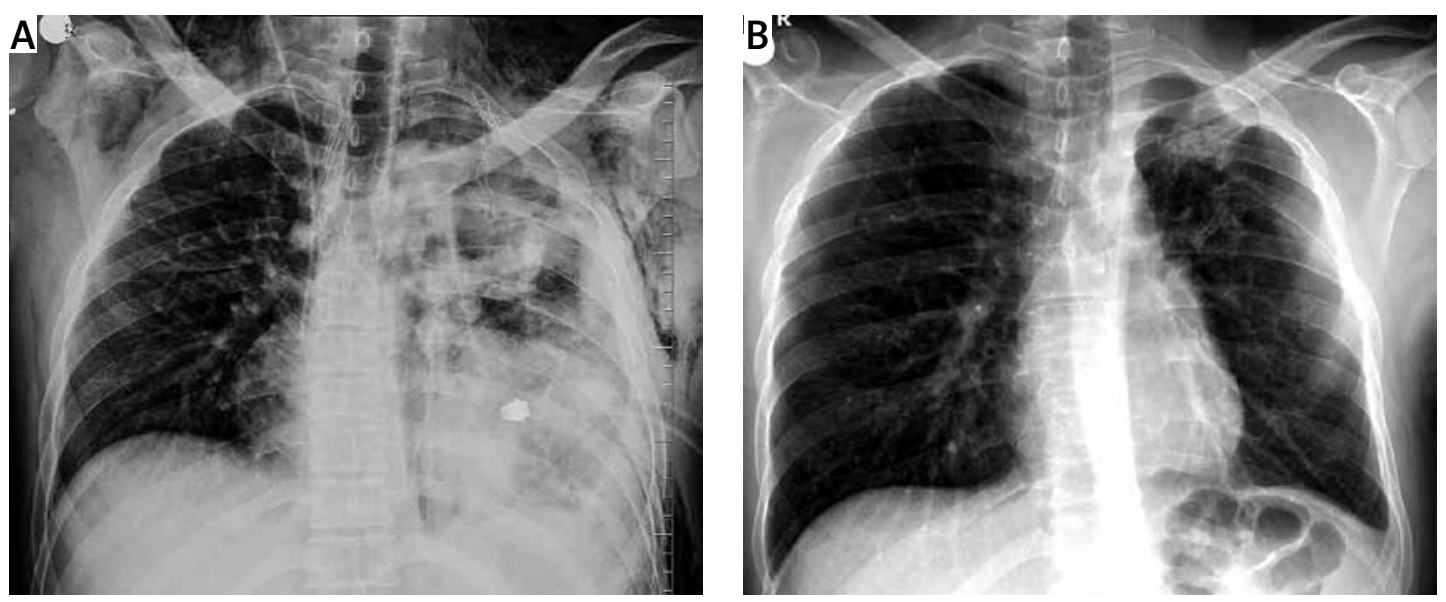

Figure 2. A - Preoperative chest X-ray. B - Postoperative chest X-ray

also contribute to this hypoxia. The treatment of both complications is mostly easy and can be rapidly eliminated by simple tube drainage. If not treated, hypoxia becomes even deeper because of the compression atelectasis is causes, and the condition of the patient may deteriorate until organ failure.

Another consequence of such penetrating injuries is intrapulmonary hematomas. This condition is mostly problem free, but it may regress spontaneously. It, however, carries the risk of embolism through bronchovenous fistulas and may be life-threatening. There is an indication of thoracotomy if an intrapulmonary hematoma causes respiratory distress and is above $5 \mathrm{~cm}$, carries an increased risk of infection, or co-exists with pulmonary abscess [3].

Another indication of thoracotomy is the wide air leakages caused by serious damage in the pulmonary parenchyma $[1,8]$. While in cases of pneumothorax in small air leakages a simple thoracic tube and closed underwater drainage would be sufficient, this mode of treatment could be insufficient in serious air leakages, and non-anatomical pulmonary resection performed by a stapler could be called for [9]. In our case, a major air leakage was detected in the left pulmonary upper lobe consistent with a gunshot wound trace when the lung was ventilated during the re-operation, and the above-mentioned resection procedure was performed with a stapler.

Another mode of treatment is damage control surgery in serious thoracic injuries such as war injuries. This may prove to be effective in enabling control of a possible intrapulmonary hematoma and the sepsis that may originate from there. Mackowski et al. demonstrated in their study that damage control surgery could be performed with reasonable results in thoracic injuries as well [10].

The duration was prolonged in our patient because of the sepsis; the general condition of the pa- tient deteriorated in time and became life-threatening. The patient received re-thoracotomy because of the above-mentioned indications, and sepsis control could only be achieved in this manner.

Consequently, thoracic injuries are the main causes of morbidity and mortality in war surgery. The fundamental problems responsible for mortality here are hemorrhage, respiratory failure, and sepsis. Following the first life-saving emergency surgery, damage control surgery or clinical evaluation of followed-up patients can be used alongside imaging methods such as X-ray, ultrasonography, and computed tomography. When a factor that may cause sepsis in the future is detected through these, early period resource control can be enabled before infection signs develop.

\section{Conflict of interest}

The authors declare no conflict of interest.

\section{References}

1. Mohan P, Mohan R. Management of warfare chest injuries. MJAFI 2010; 66: 329-32.

2. Propper BW, Gifford SM, Calhoon JH, et al. Wartime thoracic injury: perspectives in modern warfare. Ann Thorac Surg 2010; 89: 1032-5.

3. Giannou C, Baldan M. War surgery working with limited resources in armed conflict and other situations of violence. ICRC: International Committee of the Red Cross; 2010.

4. Borden Institute, Walter Reed Army Medical Center. Emergency War Surgery, Third United States Revision 2004: 143.

5. Mannion SJ, Chaloner E. Principles of war surgery. BMJ 2005; 330: 1498-500.

6. Adegboye VO, Ladipo JK, Brimmo IA, et al. Penetrating chest injuries in civilian practice. Afr J Med Med Sci 2001; 30: 327-31.

7. Leach RM, Treacher DF. Oxygen transport 2, tissue hypoxia, ABC of oxygen. BMJ 1998; 317: 1370-3.

8. Cameron JL. Current Surgical Therapy. $11^{\text {th }}$ edn. Saunders, Philadelphia 2014; 1008. 
9. Mueller MR, Marzluf BA. The anticipation and management of air leaks and residual spaces post lung resection. J Thorac Dis 2014; 6: 271-84.

10. Mackowski MJ, Barnett RE, Harbrecht BG, et al. Damage control for thoracic trauma. Am Surg 2014; 80: 910-3. 